\title{
MAGNETIC NANOPARTICLES FOR APPLICATION IN NANOMEDICINE
}

This contribution will summarize the information about the ways of synthesizing biocompatible magnetic nanoparticles and complexes containing them and the possibility of their application in nanomedicine at magnetic drug targeting and thermal treatment of diseases by hyperthermia effect. Some procedures of the preparation of biocompatible magnetizable complexes as magnetic nanoparticles, magnetic fluids, some proteins and enzymes covalently bound to the freshly prepared magnetic nanoparticles in the presence of carbodiimide (bovine serum albumin, streptokinase, chymotrypsin, dispase, glucose oxidase), entrapment of magnetic particles into magnetoliposomes and encapsulation of clinically important drug as indomethacin and taxol together with magnetite nanoparticles in biodegradable polymer. We will summarize the results from the study of structural, magnetic and hyperthermic properties of bacterial magnetite nanoparticles i.e. magnetosomes prepared by biomineralization process of magnetotactic bacteria as a promising material for application in nanomedicine.

\section{Introduction}

Since the mid 1970s, magnetic particles and fluids or ferrofluids as they are often called, have increasably been used in the area of bioscience and nanomedicine. Magnetic nanoparticles offer some attractive possibilities in biomedicine as they have controllable sizes ranging from a few nanometers up to tens of nanometers, which places them at dimensions that are smaller than or comparable to those of the cell $(10-100 \mu \mathrm{m})$, a virus $(20-450 \mathrm{~nm})$, a protein $(5-50 \mathrm{~nm})$ or a gene ( $2 \mathrm{~nm}$ wide and $10-100 \mathrm{~nm}$ long). This means that they can get close to a biological entity of interest. On the other hand, although often referred to as magnetic, many of the particles currently used are superparamagnetic, meaning that these particles can be easily magnetized with external magnetic field and redispersed immediately once the source of the magnetic field is removed. The reaction of magnetic particles to a remote magnetic force is their unique feature, which can be used in many applications involving the transport and retaining of magnetic nanoparticles with immobilized anticancer drug in targeted region of the body such as a tumor and bioseparation including cell sorting. Magnetic particles also can have resonant response to a time-varying magnetic field with advantageous results related to the transfer of energy from the exciting field to the nanoparticles. This enables effective thermotherapy (hyperthermia) as one of the promising approaches in cancer therapy. Of course, magnetic particles themselves generate a magnetic field and influence the local area around them. This feature is exploited in magnetic resonance imaging (MRI). These and many other potential applications are made available in biomedicine as a result of the special physical properties of magnetic nanoparticles. From the physiological point of view the critical parameters of magnetizable complex systems and magnetic field are particle size, surface characteristics of the particle, concentration and volume of the fluid, reversibility and strength of the drug-ferrofluid binding (desorption characteristics), access of organism (infusion route), duration and rate injection, geometry and strength of the external magnetic field. On the other hand, physiological parameters of the organism comprise size, weight, body surface, blood volume, vascular resistance, circulation time, tumor volume and location, vascular content of tumor and blood flow in tumor [1].

The principal problems currently associated with systematic drug administration are a) even biodistribution of pharmaceuticals throughout the body, b) the lack of drug specific affinity toward a pathological site, $c$ ) the necessity of a large total dose of a drug to achieve high local concentration, e) non-specific toxicity and other adverse side-effects due to high drug doses [2]. Magnetic drug targeting can bring solution to all these problems. Magnetic drug targeting allows the concentration of drugs at a defined target site generally and, importantly, away from the reticular endothelial system (RES) with the aid of a magnetic field. The intended drug and a suitable magnetically active component (magnetic fluid) are formulated into a pharmacologically stable formulation. This compound is injected intravenously in the presence of an external magnetic field with sufficient field strength and gradient to retain the carrier at the target site.

After particles are injected into the bloodstream they are rapidly coated by components of the circulation, such as plasma proteins. This process is known as opsonization and is critical in dictating the circumstance of the injected particles [3]. So, the application of nanoparticles in vivo or ex vivo would require surface

* M. Timko, P. Kopcansky, M. Koneracka, V. Zavisova, N. Tomasovicova, A. Dzarova

Institute of Experimental Physics, Slovak Academy of Sciences, Kosice, Slovakia, E-mail: timko@saske.sk 
modification that would ensure particles were non-toxic, biocompatible and stable to the reticulo-endothelial system. Generally the magnetic particles are coated by a biocompatible polymer such as polylactic acid (PLGA), derivates of dextran, polyethylene glycol (PEG), polyethylen oxide or polyoxamines. The coating acts to shield the magnetic particle from the surrounding environment and can be functionalized by attaching carboxyl group, biotin, avidin, carbodi-imide and other molecules [4-6].

\section{Biocompatible magnetic materials}

A variety of magnetic nanoparticle and microparticle carriers have been developed to deliver drugs to specific target sites in vivo. The biocompatible nanoparticles or magnetic fluids during the therapy are injected intravenously and then blood circulation would be used to transport the particles to the region of interest for treatment. Alternatively, the particle suspension would be injected directly into the treatment area. Either of these routes has the requirement that the particles do not aggregate and block their own spread. This leads to the question about the best way to produce magnetic nanoparticles and consequent magnetic fluids. It was shown that nanoparticles of about $5-10 \mathrm{~nm}$ should form the ideal particles for the most forms of therapy but that there will also be problems of formulating the particle concentrations and suspending media to obtain best distribution [7]. We will focus on some procedures to prepare a biocompatible magnetizable complex system.

\subsection{Covalent binding procedure}

The targeting of drug-bearing magnetic particles to a specific part of the body has been studied using magnetic fluids (stable suspensions of highly dispersed particles of magnetic materials), unstable suspensions (well dispersed systems containing multidomain magnetic particles) and magnetic microspheres (complex systems comprising special matrix materials like albumin, polysaccharide or containers like liposomes or erythrocytes) [8,9]. These systems are ideal for carrying small molecular weight pharmacologically active substances to a target area. However, these systems are not ideal for transporting enzymes to a target area for treatments of clot lysis in peripheral and coronary vascular occlusive diseases. In the present study, an attempt was made to link protein molecules as bovine serum albumin (BSA), glucose oxidase (GOD), chymotrypsin, streptokinase and dispase directly to magnetic particles using CDI as the coupling agent. Different values of $\mathrm{pH}$ and ratios of magnetic particles to protein were studied in order to establish the optimum conditions for immobilization. The direct coupling of enzymes or bioactive molecules to the magnetic particles has a number of potential advantages.

Several advantages could be envisaged of such preparations where molecules are directly linked to a magnetic material, like ferrite, the lack of a polymer coat results in smaller particles, thus increasing the ratio of surface area to volume, allowing a greater response to any magnetic field; studies on the use of magnetic par- ticles for cell-separation have revealed that the larger the particle size used for separation, the higher the extent of non-specific entrapment in the larger aggregates of magnetic particles, thus smaller magnetic particles hold the promise of greater specificity; small magnetic particles can exist as stable colloidal suspensions (ferrofluids) that will not aggregate, thus allowing for uniform distribution in a reaction mixture; the direct coupling method described in our papers $[6,10]$ is both simple (one-step) and inexpensive. In our experiment BSA, GOD, chymotrypsin, streptokinase and dispase were immobilized onto magnetic particles using CDI. The coupling reactions were carried out under different conditions to determine the optimum conditions for immobilization of proteins, i.e. change of the $\mathrm{pH}$ of the reaction mixture and proportion of magnetic particles to proteins. The present findings clearly show that it is possible to bind proteins onto magnetic particles in the presence of CDI without the aid of a primary coating. The binding is due to the presence of hydroxyl groups on the surface of fine magnetic particles freshly prepared from $\mathrm{Fe}_{3} \mathrm{O}_{4}$. The optimal conditions for the immobilization of the various proteins and enzymes to fine magnetic particles depend on the kind of immobilized proteins and enzymes, the $\mathrm{pH}$ of the reaction mixture and the ratio of each reagent in the reaction mixture, respectively. The present findings have several applications in medicine and biotechnology. One of the important applications worth exploring is the treatment of coronary thrombosis and peripheral arterial occlusions. The present procedure could also be used to remove carcinogenic cells from the circulation to prevent metastases by using magnetic antibodies specific for tumor cell surface antigens.

\subsection{Liposome entrapment procedure}

In order to improve the accumulation of magnetite particles in a target site the magnetoliposomes were developed [11]. This new type of vesicle consisted of nanosized magnetic particles or magnetic fluid wrapped in a phospholipids bilayer with or without drugs offers new challenges in the field of modern biotechnology and biomedicine. To overcome problem with the releasing of drug from the magnetoliposomes a variety of approaches have been employed including complexation of DNA with cationic lipids [12], the design of thermosensitive liposomes capable of releasing their contents in response to small changes in temperature [13], and the development of $\mathrm{pH}$-sensitive liposomes [14]. As the magnetoliposomes offer new challenges in the field of modern biotechnology and biomedicine it is very important to know the transport properties of the membrane-impermeant substances into cells. In our previous work [15] we have used the magnetoliposomes consisted of lipid mixture dipalmitoylphosphatidylcholine (DPPC) with nanosized magnetic particles and liposomes wrapped dye Crystal Violet for the study of transmembrane transport. The prepared magnetoliposomes were observed using Transmission Electron Microscopy. It was shown that there exists temperature dependent non-zero transport of dye from liposom to magnetoliposome. The lipid bilayer of the used DPPC provides a simplified model of cellular membrane because any "impurities" in the form of proteins are missing in the lipid bilayer. 


\subsection{Polymer nanospheres}

Magnetic particles encapsulated with drug into biocompatible polymer have potential applications in the field of magnetic drug targeting. Non-specific intravenously administered drugs are systemically distributed and attack normal, healthy cells in addition to the target cells. Magnetically controlled drug targeting could reduce side effects. This technique is based on the binding of a selected drug with magnetic fluids into a pharmacologically stable formulation. The drug can be dissolved, entrapped, encapsulated or attached to nanoparticles and depending on the method of preparation, nanoparticles, nanospheres (NPs) or nanocapsules can be obtained. Biodegradable NPs formulated using biodegradable polymer poly(lactide-co-glycolide) (PLGA), can be used as carriers for drug. In addition to being biodegradable, other advantages include reduced frequency of administration, sustained drug release, reduced dosage, and less systemic side effects [16]. Polylactic acid (PLA) and PLGA have been approved for human use by the United States Food and Drug Administration (USFDA) as surgical sutures, implantable devices, and drug delivery systems [17]. Magnetic-polymeric NPs (MNPs) must fulfill several requirements such as biocompatibility, biodegradability, mechanical strength, and ease of producing. The constituents of the magnetically active polymeric NPs play different roles: the polymeric matrix acts as a shell, reservoir, and vehicle for the active component, whereas magnetite is the component which makes targeting possible by external magnetic field manipulation. Well known classes of biodegradable materials for controlled release are the PLGAs. The main advantage of these degradable polymers is that they are broken down into biologically acceptable molecules that are metabolized and removed from the body via normal metabolic pathways.

Indomethacin (IND) is a nonsteroidal anti-inflammatory drug (NSAID) that reduces fever, pain, and inflammation. As IND may cause or worsen stomach or intestinal bleeding or ulcers and can increase blood pressure and decrease kidney function, it is important to decrease used dosages and side effects during the treatment. One of the possibilities to do it is direct delivery of the drug to the target area of the body by external magnetic field. Magnetically controlled drug targeting is one of the various possibilities of drug targeting. This technology is based on the encapsulation of drug (in our case IND) with magnetic particles that concentrate the drug in the area of interest by means of magnetic fields.

In our previous study $[18,19]$ IND, which is a poorly watersoluble antiinflammatory drug, was encapsulated in magnetic biodegradable PLA. The magnetite nanoparticles were prepared by the coprecipitation method of ferric and ferrous salts in an alkali aqueous medium was used to prepare magnetite particles [15] Sodium oleate $\left(\mathrm{C}_{17} \mathrm{H}_{33} \mathrm{COONa}\right)$ as surfactant was used for the modification of prepared magnetic particles to prevent their agglomeration. Sodium oleate $\left(10 \mathrm{wt} \%\right.$ with respect to $\mathrm{Fe}_{3} \mathrm{O}_{4}$ ) was added to the suspension of $\mathrm{Fe}_{3} \mathrm{O}_{4}$ and mixed under heating until the boiling point was reached. The saturation magnetization of the prepared magnetic fluid (with a $2 \%$ volume concentration of $\mathrm{Fe}_{3} \mathrm{O}_{4}$ ) and average diameter of magnetite particles were estimated by VSM magnetometer to be $6.5 \mathrm{emu} / \mathrm{g}$ and $10 \mathrm{~nm}$, respectively.
Infrared spectroscopy confirmed the incorporation of magnetic particles and drug in the polymer. The next objectives were focused on the enhancement of incorporation efficiency of drug including the influence of the $\mathrm{pH}$ aqueous phase in magnetic PLA nanospheres. Finally, the release behavior of IND was examined by an external sink method. Two simple models, diffusion and dissolution, were applied for the description of the experimental data of IND release and for the identification of the release mechanism. It can be said that the encapsulation of IND into magnetite-labeled biodegradable polymer was successfully realized. The biodegradable polymer nanoparticles made by nanoprecipitation method were spherical in shape, and their particle size was about $250 \mathrm{~nm}$ in diameter. The successful encapsulation of the IND and magnetic particles into PLA was confirmed by infrared spectroscopy. Release experiments showed that $70 \%$ of the drug was released within $5 \mathrm{~h}$ and a plateau was reached within $10 \mathrm{~h}$ at $80 \%$. Such IND-loaded magnetic nanoparticles could be useful for magnetic drug targeting of inflammatory diseases.

Taxols (paclitaxel, TAX) an important anticancer drug, has been chosen for encapsulation into the polymer for its significant role against a wide range of tumors (breast cancer, ovarian carcinoma, lung cancer, and head and neck carcinomas) [20]. TAX is a natural diterpenoid alkaloid, firstly isolated from the bark of the yew Taxus brevifolia [21]. It is not affordable from the nature. The other limitation of TAX applications is its high insolubility in water and most pharmaceutical solvents. Adjuvants such as Cremophor EL have to be used in its current clinical administration, which, by itself, causes serious side effect hypersensitivity reaction. To eliminate the side effects of the adjuvant and to improve the therapeutic efficacy of the drug, alternative dosage forms have been suggested, including liposomes, microspheres, and polymeric nanoparticles (NPs). Other important advantages associated with the use of NPs include the simplicity of their preparation with welldefined biodegradable polymers and their high stability in biological fluids and during storage. The modified nanoprecipitation method was used to entrap magnetic fluid (MFPEG) and anticancer drug TAX into polymer NPs. To prepare a stable colloid of magnetic particles, sodium oleate $\left(\mathrm{C}_{17} \mathrm{H}_{33} \mathrm{COONa}\right)$ as a first surfactant was used to prevent their agglomeration. The key parameters of the behavior of magnetic particles are related to the nature of their surface. The chemical composition of the surface is especially important to avoid the action of the reticuloendothelial system (RES), which is a part of the immune system, to increase the halflife in the blood stream. Coating the magnetic particles with a neutral and hydrophilic compound-poly(ethylene glycol), the circulatory half-life increases from minutes to hours or days. Thus, PEG as a second surfactant was added to the system magnetite-sodium oleate. The more detail description of preparation, procedure, magnetic and morphological properties is given in our papers [22, 23]. Scanning Electron Microscopy (SEM) was used to determine the morphology and size of various TAX-loaded nanoparticles (TAXMNPs shown in Fig. 1). The surface was primarily smooth, although some roughness could be identified in certain areas of some spheres. The mean diameter of all NP samples was approximately 200-250 $\mathrm{nm}$ and the results were in good agreement with the results obtained with the PCCS technique. 


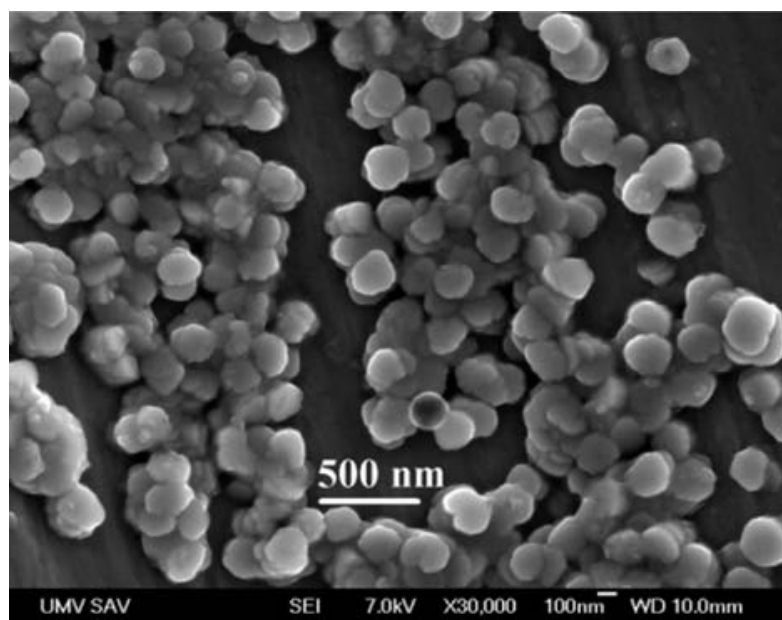

Fig. I SEM image of taxol-magnetite-PLGA nanospheres

We can state that we have prepared and characterized TAXMNPs with a spherical shape of the mean diameter lower than $250 \mathrm{~nm}$, which is a relevant size for intravenous administration. Using IR spectroscopy, TAX was successfully identified in the TAXMNPs before the release process and also in the TAXMNPs that had been releasing TAX into a phosphate buffer. The prepared NPs have been found to have good stability in the presence of high $\mathrm{NaCl}$ concentration at ambient temperature, the toxicity (preliminary 'up and down' method) of prepared samples declared a three times higher value of lethal dose LD50 (see Table 1) in comparison with pure TAX (LD50 $=33 \mathrm{mg} / \mathrm{kg}$ ) and showed a significant response to the external magnetic field which is useful for drug delivery systems for tumor treatment (see Fig. 2).

The lethal dose in vivo experiments

Table 1

\begin{tabular}{|c|c|}
\hline Sample & LD50 \\
\hline MFPEG & $400 \mathrm{mg} \mathrm{Fe} 3 \mathrm{O} / \mathrm{kg}$ \\
\hline PLGA NPs & $221 \mathrm{mg} \mathrm{PLGA} / \mathrm{kg}$ \\
\hline PLGA/TAX & $\begin{array}{c}226 \mathrm{mg} \text { PLGA } \mathrm{kg} \\
\text { (at } 11.3 \mathrm{mg} \mathrm{TAX} / \mathrm{kg} \text { ) }\end{array}$ \\
\hline PLGA/MFPEG & $174-198 \mathrm{mg} \mathrm{PLGA} / \mathrm{kg}$ \\
\hline PLGA/MFPEG/TAX & $\begin{array}{c}154 \mathrm{mg} \text { PLGA } / \mathrm{kg} \\
\text { (at } 7.7 \mathrm{mg} \mathrm{TAX} / \mathrm{kg} \text { ) }\end{array}$ \\
\hline
\end{tabular}

A good sink condition was achieved using sodium salicylate solution, a hydrotropic agent, as a good alternative to increase the aqueous TAX solubility for in vitro TAX release studies from TAXMNPs. The effect of $\mathrm{pH}(\mathrm{pH}=6.0,6.6$, and 7.4) as well as the molar concentration of sodium salicylate in the release medium $(1,2$, and $3 \mathrm{M})$ on the release profiles of drug were studied and it can be concluded that TAX release from TAXMNPs was not significantly influenced by the $\mathrm{pH}$ value of the phosphate buffer (Fig. 3 ). The slope of the initial release process increased with increasing sodium salicylate concentration of the release medium. The presence of sodium salicylate in the release medium significantly influ-

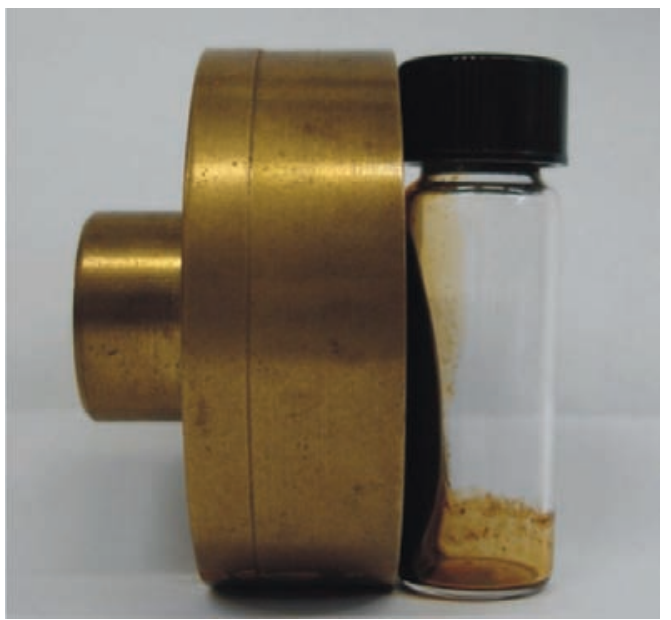

Fig. 2 Encapsulated taxol and MF-PEG in PLGA attached to a magnet

enced the release profile of TAX and the cumulative released amount of TAX in 9 days was around $50 \%$ (for 2 and $3 \mathrm{M}$ sodium salicylate).

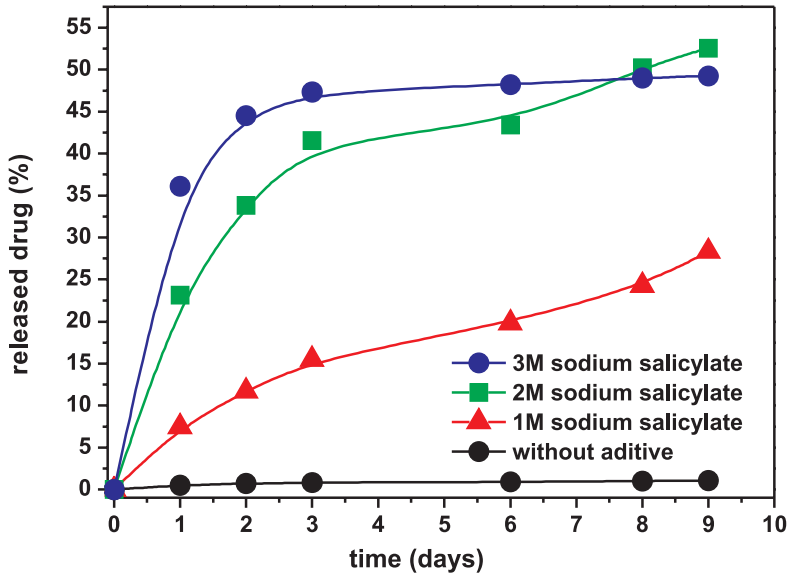

Fig. 3 Time dependence of the released drug at $p H=7.4$ from TAXMNPs to (a) phosphate buffer, (b) phosphate buffer with $1 M$ sodium salicylate, (c) phosphate buffer with $2 \mathrm{M}$ sodium salicylate and (d) phosphate buffer with $3 M$ sodium salicylate

The surface modification of magnetite with PEG was a useful approach to prepare biocompatible magnetic fluid suitable for entrapment into a hydrophobic polymer PLGA together with the anticancer drug taxol by the nanoprecipitation method. The nanospheres were nearly spherical with the mean diameter of $250 \mathrm{~nm}$ and with entrapment efficiency of magnetite $21.5 \mathrm{wt} \%$ and taxol $0.5 \mathrm{wt} \%$. They were superparamagnetic, with saturation magnetization of $1.4 \mathrm{mT}$. Nevertheless, the prepared taxol loaded magnetic polymeric nanospheres still show sufficient magnetization for their magnetic properties to be useful from the point of view of magnetic carrier technology. The prepared TAXMNPs were used in in-vivo 
experiments at treatment of melanoma cancer B16 in mouse. We found that after intravenous application of prepared TAXMNPs with application of the external magnetic field statistical important reduction of melanoma was observed.

\subsection{Biological magnetite nanoparticles - magnetosomes}

Application of magnetic materials for hyperthermia treatment of biological tissues with the goal of tumor therapy has been known in principle for more than four decades [24]. The heating effect depends strongly on the magnetic properties of the particles, which may vary appreciably depending on their size and microstructure. The magnetite particles can be either ferromagnetic or superparamagnetic, and the magnetic moment of single-domain particle relaxes through either Brownian or Neel relaxation depending on the anisotropy and size of the particle [25, 26]. It was shown by previous investigations that specific loss power (SLP) depends strongly on the mean particle size as well as the width of the size distribution [27-29]. The strong monotonous rise of SLP, nearly two orders of the magnitude, from $15 \mathrm{~kW} / \mathrm{kg}$ up to $900 \mathrm{~kW} / \mathrm{kg}$ with an increasing particle core size from $7 \mathrm{~nm}$ up to $18.4 \mathrm{~nm}$ in magnetite, was described. This rise was clarified in the frame of the classical Debye theory of dispersions [28]. For the biocompatible magnetic iron oxides magnetite and maghemite, a maximum SLP was found above typical sizes of superparamagnetic particles but below the size of typical multidomainparticles. On the other hand, the SLP determined from hysteresis loops, susceptibility spectra and calorimetry with a maximum value of $960 \mathrm{~kW} / \mathrm{kg}$ at $410 \mathrm{kHz}$ and field amplitude of $10 \mathrm{kA} / \mathrm{m}$ was found for biological particles-magnetosomes with a mean size of about $35 \mathrm{~nm}$ [30]. However, the magnetic and structural properties of magnetosomes have been insufficiently characterized up to now, mainly because of the unavailability of significant amounts of material. Magnetosomes, which can be obtained by a biomineralization process in magnetotactic bacteria (MTB), consist of magnetic mineral crystals magnetite or greigite [31] enveloped by a biological membrane that contains phospholipids and specific proteins [32]. The magnetosome membrane is not only critical for the control of crystal size and morphology, but it also prevents the aggregation of extracted magnetosomes and thus stabilizes magnetosome suspensions. Magnetosome crystals of MTB are typically from 30 to about $140 \mathrm{~nm}$ in diameter, i.e. within the single-magnetic-domain size range, which maximizes the efficiency of the particle as a permanent magnetic carrier [33]. In many magnetotactic bacterial types, the magnetosome are characterized by narrow particle size distributions. Whether the mineral particles are magnetite or greigite, the chain of magnetosome particles constitutes a permanent magnetic dipole fixed within the bacteria [34]. The remanent moment is generally close to its saturation value. Normally it is sufficiently larger than background thermal energy so that it, and consequently the bacteria, is oriented along geomagnetic field lines as it swims, causing the bacterium to migrate along the field lines. Because of their unique characteristics, magnetosomes have a high potential for nano- and biotechnological applications, which require a specially designed particle surface. Especially, in biotechnologica applications, functionalized bacterial magnetosomes represent an attractive alternative to chemically synthesized iron oxide particles. Magnetosome particles have been applied in numerous applications that range from the extraction of magnetic resonance imaging (MRI), magnetic drug targeting, DNA and RNA to the highly sensitive detection and concentration of toxic substances and development of immunoassays [35]. One of the potential application areas of magnetosomes is magnetic particle hyperthermia (MPH) [36]. As pointed out recently an enhancement of specific heating power is of importance for reducing useful dosage applied to the tumor. Previous investigations on the suitability of magnetic nanoparticles for MPH have shown that for the biocompatible magnetic iron oxides a core size range above about $20 \mathrm{~nm}$ is advantageous with respect to large specific heating power. Therefore, magnetosomes are of particular interest for testing their suitability for application in MPH tumor therapy.

\section{Magnetic hyperthermia effect}

In our previous papers $[37,38]$, the structural, magnetic properties and heating effect of magnetosomes obtained by a biomineralization process in magnetotactic bacteria Magnetospirillum sp. Strain AMB-1 were reported. The heating effect of the magnetosome solution as the result of absorbing energy from the alternating magnetic field has shown that magnetosome suspensions are capable of delivering sufficient heating power which may be of interest for magnetic particle hyperthermia.

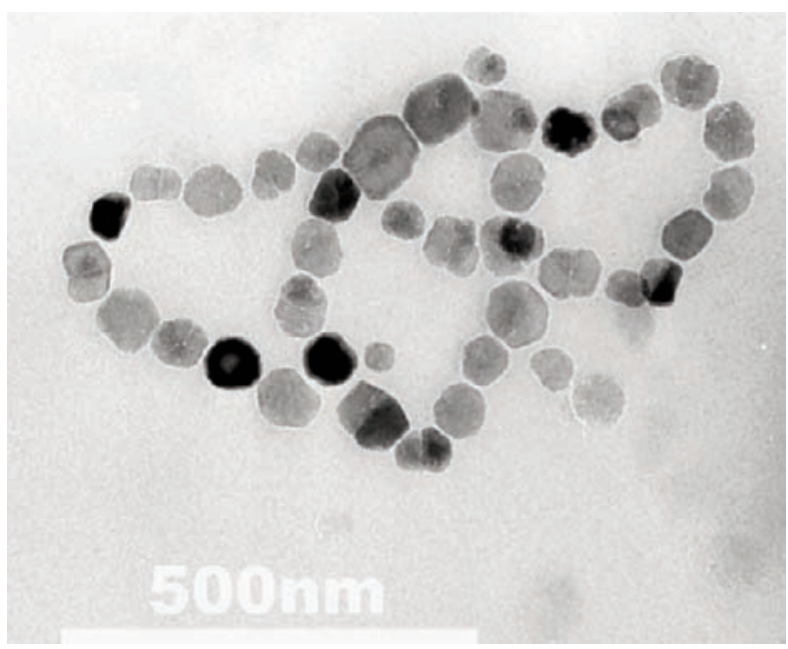

Fig. 4 Transmission electron micrograph of magnetosomes

Bacterial magnetosomes investigated in this contribution were synthesized by magnetotactic bacteria Magnetospirillum sp. strain AMB-1 in laboratory conditions. These bacteria are Gram-negative a-proteobacterium that are more oxygen-tolerant and easier to grow on a large scale. The detailed description of cultivation of magnetotactic bacteria and isolation of magnetosomes is given in our previous contribution [39]. Techniques for the isolation and purification of magnetosome particles from Magnetospirillum sp. are based on combination of centrifugation and the magnetic sep- 
aration. Typically, $2.6 \mathrm{mg}$ bacterial magnetite could be acquired from a 1000-mL culture of Magnetospirillum sp. AMB-1. These isolation and purification procedures leave the surrounding membrane intact and magnetosome preparations are apparently free of contaminating materials. Owing to the presence of the enveloping membrane, isolated magnetosomes particles form stable, well dispersed suspensions in water solution of HEPES (4-(2-hydroxyethyl)-1-piperazineethanesulfonicacid). For the study of heating characteristics of samples with magnetosomes we used the heating system described in [18] at the frequency of $750 \mathrm{kHz}$ and ACfield amplitude in the range of $(0-2.5) \mathrm{kAm}^{-1}$. The slope of the curve $T(t)$ is a measure of the power release in a unit volume. From the fitting of the function $(\Delta T / \Delta t)=(H / a)^{n}$ to the experimental data the parameters $a$ and $n$ were determined which depend on several factors such as particle permeability, conductivity, size, shape and distribution. The observed $H^{n}$-law-type dependence of the temperature increase rate $(\Delta T / \Delta t)_{t=0}$, on the amplitude of the magnetic field indicates the presence of superparamagnetic and partially ferromagnetic particles in the magnetic fluids studied since $n>2$. The small amount of ferromagnetic particles causes energy losses associated with hysteresis and superparamagnetic particles cause energy losses associated with relaxation. On the basis of the obtained relation $(\Delta T / \Delta t)_{t=0}=(\mathrm{H} / 14063)^{2.31}$ the specific absorption rate $(S A R)$ values were calculated. The $S A R$ is defined as the amount of heat released by a unit weight of the material per unit time. It can be calculated from the expression $S A R_{\text {sample }}=C_{s}(\Delta T / \Delta t)\left[\mathrm{mW} / \mathrm{g}_{\text {sample }}\right]$ where $C_{s}$ is the specific heat of the sample. The SAR data normalized with respect to the magnetite mass contents in the samples, $m_{\mathrm{Fe}}$ can be calculated from the expression:

$$
S A R=\frac{\rho C_{P}}{m_{\mathrm{Fe}}} \cdot\left(\frac{\Delta T}{\Delta t}\right)_{t=0}\left[\frac{\mathrm{W}}{\mathrm{kg}_{\mathrm{Fe}}}\right]
$$

where $\rho$ is the density of the sample, $C_{P} \cong C_{\text {water }}=4.18\left[\mathrm{~kJ} \cdot \mathrm{K}^{-1}\right.$. $\left.\cdot \mathrm{kg}^{-1}\right]$ is the sample specific heat capacity, $m_{\mathrm{Fe}}=\phi V \cdot \rho_{\mathrm{Fe}}=21$ $\left[\mathrm{kgFe} \cdot \mathrm{m}^{-3}\right.$ sample] is the mass of magnetic material in the $1 \mathrm{~m}^{3}$ sample and $\rho_{\mathrm{Fe}}=5180\left[\mathrm{~kg} \cdot \mathrm{m}^{-3}\right]$ is the density of magnetite.

Densities $\rho_{0}$ and $\rho$ of the carrier liquid and the

Table2 magnetosome-based magnetic fluid, respectively, mass contents of magnetite in the samples, $m_{\mathrm{Fe}}$, and the values of the parameters a and $n$ determined from thermal measurements.

\begin{tabular}{|c|c|c|c|c|c|}
\hline$\rho_{0}$ & $\rho$ & $\Phi_{V}$ & $m_{\mathrm{Fe}}$ & $\mathrm{a}$ & $\mathrm{N}$ \\
\hline $\mathrm{kg} \cdot \mathrm{m}^{-3}$ & $\mathrm{~kg} \cdot \mathrm{m}^{-3}$ & $\%$ & $\mathrm{~kg} \cdot \mathrm{m}^{-3}$ & - & - \\
\hline 998 & 1002 & 1.1 & 21 & 12162 & 2.31 \\
\hline
\end{tabular}

On the basis of the relation, $(\Delta T / \Delta t)_{t=0}=(\mathrm{H} / 12162)^{2.31}$, and the following expression for the power dissipated as heat in $1 \mathrm{~g}$ of magnetic material (magnetosome) was obtained:

$$
S A R=201 \cdot\left(\frac{H}{12162}\right)^{2.31}\left[\frac{\mathrm{kW}}{\mathrm{kg}_{F e}}\right]
$$

The dependence of SAR on the magnetic field with respect to the magnetite mass content in the sample calculated according Eq. (1) (at $f=750 \mathrm{kHz}$ ) is shown in Fig. 5. The found value for a specific absorption rate (SAR) $26 \mathrm{~kW} / \mathrm{kg}$ at $5 \mathrm{kA} / \mathrm{m}$ and $750 \mathrm{kHz}$, which is comparable to those values of the MF-oleate system means that magnetosomes may be considered as good material for the biomedical applications in hyperthermia in the view of a phospholipidic biological membrane.

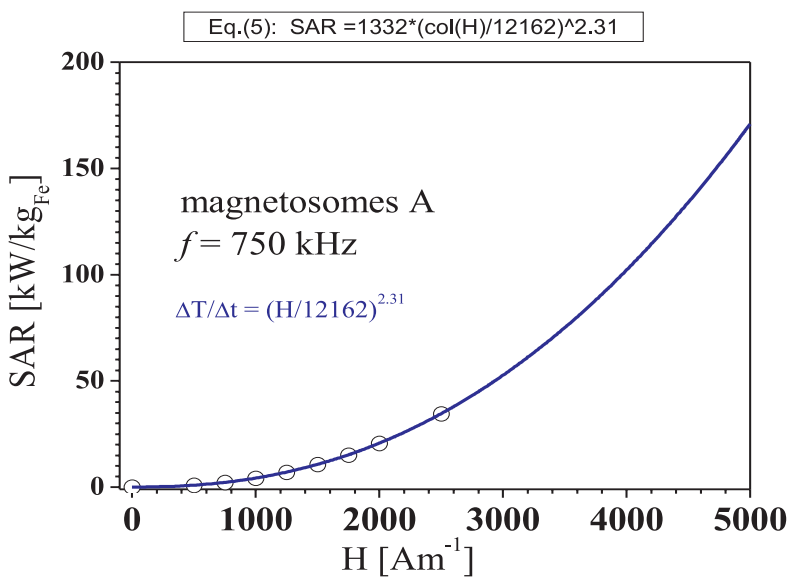

Fig. 5 SAR values for the sample at $f=750 \mathrm{kHz}$ calculated with the aid of $E q(2)$

The obtained results in the area of biomedical applications are very promising but there are several problems associated with magnetic drug targeting. These limitations include (i) the possibility of embolization of the blood vessel in the target region due to the accumulation of magnetic carriers, (ii) difficulties with scaling up from animal models due to larger distances between the target sites and the magnet, (iii) once drug is released, it is no longer attracted to the magnetic field and (iv) toxic responses to the magnetic carriers [31]. Recent pre-clinical and experimental results indicate, however, that it is still possible to overcome these limitations and use magnetic targeting to improve drug retention and also address safety issue.

\section{Conclusion}

In this contribution we have showed some aspects of preparation biocompatible magnetizable complex systems (nanoparticles, magnetic fluids, covalently binding of proteins and enzymes to magnetite nanoparticles, magnetoliposomes, encapsulation clinically important drugs (Indomethacin and Taxol) in biodegradable polymer nanospheres together with magnetite and their in vivo application, preparation and properties of biological magnetite nanoparticles - magnetosomes and their application in hyperthermia experiment. Greater understanding of the properties of magnetic particles and overcoming of some problems connected with biomedical application will enable the earlier implementation of laboratory results into clinical praxis. 


\section{Acknowledgement}

The Slovak Academy of Sciences, in the framework of CEX Nanofluid, Projects VEGA 077 and 051, Project SAV-FM-EHP2008-01-01 and the Slovak Research and Development Agency, in the framework of Projects APVV No. 0173-06, No. 0509-07 and Ministry of Education Agency for structural funds of EU in framework of projects No. 26220120021 and 26220220005.

\section{References}

[1] LUBE, A. S., BERGEMANN C. et al.: Cancer Res. 194 (1999) 149

[2] TORCHILIN, V. P.: Eur. J. Pharm. Sci. 11 Suppl. 2 (2000) S81

[3] DAVIS, S. S.: Trends Biotechnol., 15 (1977) 217

[4] MECHTA, R. V., UPHADAY, R. V., CHARLES, S.W., RAMCHAND, C. N.: Biotechnol. Techn. 11, (1997) 493

[5] KONERACKA, M., KOPCANSKY, P., TIMKO, M., ANTALIK, M., RAMCHAND, C. N., LOBO, D., MECHTA, R., UPHADAY, R. V.: J. Magn. Magn. Mat. 201 (1999) 427

[6] KONERACKA, M., KOPCANSKY, P., TIMKO, M., RAMCHAND, C. N., DE SEQUEIRA, A., TREVAN, M.: J. Mol. Catalysis B Enzymatic 18 (2002) 13

[7] BERRY, C. C., CURTIS, A. S. G.: J. Phys. D: Appl. Phys. 36 (2003) R198

[8] BACRI, J. C., SALIN, D., MASSART, R.: J. Magn. Magn. Mater. 85 (1990) 27.

[9] PACKER, L., TRISTRAM, S., HERZ, J. M., RUSSEL, C., BORDERS, L. C.: FEBS Lett. 108 (1979) 243

[10] ZAVISOVA, V., KONERACKA, M., TOMASOVICOVA, N., KOPCANSKY, P., TIMKO, M.: Z. Phys.Chem. 220 (2006) 241

[11] DE CUYPER, M., JONIAU, M.: Langmuir 7 (1991) 647

[12] XU, Y., SZOKA, F. C.: Biochemistry 35 (1996) 5616.

[13] GABER, M. H., HONG, K., HUANG, S. K., PAPAHADJOPOULOS, D.: Pharm Res 12 (1995)1407

[14] MEYER, O., PAPAHADJOPOULOS, D., LEROUX, J-C.: FEBS Lett 421 (1998) 61

[15] KONERACKA, M., KOPCANSKY, P., SOSA, P., BAGELOVA, J., TIMKO, M.: J. Magn. Magn. Mater. 293 (2005) 271

[16] HICKEY, T., KREUTZER, D., BURGESS, D. J., MOUSSY, F.: Biomaterials 23 (2002) 1649.

[17] MIDDLETON, J. C., TIPTON, A. J.: Biomaterials 21 (2000) 2335.

[18] TIMKO, M., KONERACKA, M., KOPCANSKY, P., TOMASOVICOVA, N., ZAVISOVA V.: J. Magn. Magn. Mat. 300 (2006) e191-e194

[19] ZAVISOVA, V., KONERACKA, M., STRBAK, O., TOMASOVICOVA, N., KOPCANSKY, P., TIMKO, M., VAVRA, I.: J. Magn. Magn. Mat. 311 (2007) 379-382

[20] FONSECA, C., SIMES, S., GASPAR, R.: J. Control. Rel. 83 (2002) 273.

[21] WANI, M. C., TAYLOR, H. L., WALL, M. E. et al.: J. Am. Chem. Soc. 93 (1971) 2325

[22] KONERACKA, M., MUCKOVA, M., ZAVISOVA, V., TOMASOVICOVA, N., KOPCANSKY, P., TIMKO, M., JURIKOVA, A., CSACH, K., KAVECANSKY, V., LANCZ, G.: J. Phys.: Condens. Matter 20 (2008) 204151

[23] KONERACKA, M., MUCKOVA, M., ZAVISOVA, V., TOMASOVICOVA, N., KOPCANSKY, P., TIMKO, M., LANCZ, G., PATOPRSTA, B., BARTOS, P., FABIAN, M.: J. Mag. Mag. Mat. 321 (2009) 1613

[24] CHAN, D. C. F., KIRPOTIN, D. B., BUNN, P.A. Jr.: J. Magn. Magn. Mater. 122 (1993) 374.

[25] JORDAN, A., SCHOLZ, R., WUST, P., SCHIRRA, H. et al.: J. Magn. Magn. Mater. 194 (1999) 185.

[26] ROSENSWIEG, R. E.: J. Magn. Magn. Mater. 252 (2002) 370.

[27] ATSUMI, T., JEYADEVAN, B., SATO, Y., TOHJI, K.: J. Magn. Magn. Mater. 310 (2007) 2841.

[28] HERGT, R., HIERGEIST, R., ZEISBERGER, M., GLOCKL, G., WEITSCHIES, W., RAMIREZ, L. P., HILGERD, I., KAISER, W.A.: J. Magn. Magn. Mater. 280 (2004) 358.

[29] HERGT, R., HIERGEIST, R., HILGER, I., KAISER, W.A., LAPATNIKOV, Y., MARGEL, S., RICHTER, U.: J. Magn. Magn. Mater. 270 (2004) 345.

[30] HERGT, R., HIERGEIST, R., ZEISBERGER, M., SCHULER, D., HEYEN, U., HILGER, I., KAISER, W.A.: J. Magn. Magn. Mater. 293 (2005) 80.

[31] FRENKEL, R. B., BLAKEMORE, R.: Science 203 (1979) 1355.

[32] SCHULER, D.: Arch. Microbiol. 181 (2004) 1.

[33] BUTLER, R. F., BANERJEE, S.: J. Geophys. Res. 80 (1975) 4049

[34] FRENKEL, R. B.: Ann. Rev. Biophys. Bioeng. 13 (1984) 85

[35] SCHULER, D.: J. Molec. Microbiol. Biotechnol. 1 (1999) 79.

[36] MOROZ, P., JONES, S. K., GRAY, B. N.: Int. J. Hyperthermia 18 (2002) 267.

[37] TIMKO, M., DZAROVA, A., KOVAC, J., KOPCANSKY, P., GOJZEWSKI, H., SZLAFEREK, A.: Acta Physica Polonica 115 (2009) 381

[38] TIMKO, M., DZAROVA, A., KOVAC, J. et.al.: J. Mag. Mag. Mat. 321 (2009) 1521

[39] TIMKO, M., DZAROVA, A., ZAVISOVA et. al.: Magnetohydrodynamics Journal 44 (2008) 3. 